\title{
Duality Principle for Image Regularization and Perceptual Color Correction Models
}

\author{
Thomas Batard and Marcelo Bertalmío * \\ Department of Information and Communication Technologies \\ Universitat Pompeu Fabra, Barcelona, Spain \\ \{thomas . batard, marcelo. bertalmio\}@upf .edu
}

\begin{abstract}
In this paper, we show that the anisotropic nonlocal total variation involved in the image regularization model of Gilboa and Osher [15] as well as in the perceptual color correction model of Bertalmío et al. [4] possesses a dual formulation. We then obtain novel formulations of their solutions, which provide new insights on these models. In particular, we show that the model of Bertalmío et al. can be split into two steps: first, it performs global color constancy, then local contrast enhancement. We also extend these two channel-wise variational models in a vectorial way by extending the anisotropic nonlocal total variation to vector-valued functions.
\end{abstract}

Keywords: Nonlocal variational problem; Duality principle; Contrast enhancement; Regularization; Perception

\section{Introduction}

Regularizing an image and modifying its contrast are very useful tasks for correcting defects produced during the acquisition process of a real-world scene and its reproduction on a display, due to physical and technological limitations (see e.g. [3] for more details). These tasks are also useful as pre-processing stage in order to improve the results of higher level applications like pattern recognition.

Variational methods are a very powerful tool for performing regularization and contrast modification of an image, and have been extensively used over the last two decades. Regarding contrast modification, the pioneer variational approach is due to Sapiro and Caselles [23], who performed contrast enhancement for histogram equalization purpose. Since then, this variational formulation has been generalized and applied in different contexts: perceptual color correction [4],[20], tone mapping [14], and gamut mapping [25] to name a few. Connections have also been made between the variational formulation [4] and Retinex theory [5],[6]. Concerning regularization tasks, the Rudin-Osher-Fatemi (ROF) denoising model [22] based on minimizing the total variation of an image has influenced

\footnotetext{
* This work was supported by the European Research Council, Starting Grant ref. 306337, by the Spanish government, grant ref. TIN2012-38112, and by the Icrea Academia Award.
} 
many local variational approaches that use generalizations of the total variation (see e.g. [2],[7],[8],[16]). On the other hand, since the seminal nonlocal approaches of Buades et al. [9], and Awate et al. [1] for image denoising, several nonlocal variational formulations for image regularization have been proposed (see e.g. [15],[17],[18]). In particular, Gilboa and Osher [15] introduced the concept of nonlocal gradient operator from which they derive a nonlocal total variation.

Computing the solutions of the variational problems aforementioned is not straightforward, mainly due to their non differentiability that makes the solutions not reachable through standard gradient descent algorithms. The situation is even more tricky when dealing with the contrast modification variational models $[4],[20]$ since they are not convex. The use of duality principle in convex optimization (see e.g. [21] for an introduction) has been a breakthrough for solving non differentiable variational convex problems in imaging. For instance, Chambolle [11] made use of the duality principle for solving the Rudin-OsherFatemi model [22]. This approach had two assets compared with the original approach of Rudin, Osher and Fatemi. First, it provides a better approximation of the solution since it does not require a regularization of the variational model. Secondly, it provides a better insight on the model by showing that this latter consists in removing oscillating patterns of the original image, which includes noise but also texture as showed by Meyer [19].

In [15], Gilboa and Osher proposed two nonlocal variational models for image regularization based on two nonlocal extensions of the total variation, called isotropic and anisotropic (nonlocal) total variation, and in this paper we point out that the anisotropic total variation corresponds to the contrast modification term in the perceptual color correction model of Bertalmío et al. [4]. Whereras Gilboa and Osher propose to solve the corresponding variational formulation with graph-cut techniques and Bertalmío et al. with a gradient descent associated to a regularized version of the variational model, none of them investigated whether the variational model possesses a dual formulation, and the main contribution of this paper is to show that both do possess a dual formulation. Other contributions are the reinterpretation of their solutions from the proposed dual formulations as well as the vectorial extension of these models by extending the anisotropic nonlocal total variation to vector-valued functions.

The outline of this work is as follows. We first show in section 2 that the anisotropic nonlocal total variation and its vectorial extension possess a dual formulation, from which we derive in section 3 dual formulations of both variational models and novel formulations of their solutions. Finally, we show in section 4 that the novel formulation of the solution of the perceptual color correction model provides a new insight on this model.

\section{Dual formulation of the anisotropic nonlocal vectorial total variation}

Definition 1 (Non local gradient). Let $\Omega$ be a compact subset of $\mathbb{R}^{2}$ and $u: \Omega \longrightarrow \mathbb{R}^{n}$ be an $n$-channel image. We assume that $\mathbb{R}^{n}$ is equipped with a 
definite positive quadratic form $h$.

A nonlocal gradient is an operator $\nabla_{w}^{N L}: C^{\infty}\left(\Omega ; \mathbb{R}^{n}\right) \longmapsto C^{\infty}\left(\Omega \times \Omega ; \mathbb{R}^{n}\right)$ of the form

$$
\nabla_{w}^{N L} u:(x, y) \longmapsto w(x, y)(u(y)-u(x))
$$

and $w: \Omega \times \Omega \longrightarrow \mathbb{R}^{+*}$ is a smooth symmetric function.

Note that definition (1) is nothing but the vectorial extension of the nonlocal gradient introduced by Gilboa and Osher [15].

Standard choices for the weight function $w$ are Euclidean distance, Gaussian kernel, and patch-based distances. We refer to Zosso et al. [26] for a weight function specific to color images determined by the hue difference between two colors. The choice of the definite positive quadratic form $h$ greatly depends on the nature of the image to be processed. Dealing with color images, a suitable choice for $h$ is the perceptual metric associated to the color space involved (e.g. the Euclidean metric associated to the CIE Lab color space).

Definition 2 (The space $W_{1, p}^{N L}\left(\Omega ; \mathbb{R}^{n}\right)$ ). The quadratic form $h$ induces a scalar product $\langle$,$\rangle on C^{\infty}\left(\Omega \times \Omega ; \mathbb{R}^{n}\right)$ defined by

$$
\left\langle\eta_{1}, \eta_{2}\right\rangle:=\int_{\Omega \times \Omega}\left(\eta_{1}(x, y), \eta_{2}(x, y)\right)_{h} d x d y
$$

where $(,)_{h}$ denotes the scalar product with respect to $h$.

The $L^{p}$ norm on $C^{\infty}\left(\Omega \times \Omega ; \mathbb{R}^{n}\right)$ is defined by

$$
\|\eta\|_{L^{p}}:=\left(\int_{\Omega \times \Omega}\|\eta(x, y)\|_{h}^{p} d x d y\right)^{1 / p}
$$

where \|\|$_{h}$ denotes the norm associated to $h$. In particular, we have

$$
\left\|\nabla_{w}^{N L} u\right\|_{L^{p}}=\left(\int_{\Omega \times \Omega}\|w(x, y)(u(y)-u(x))\|_{h}^{p} d x d y\right)^{1 / p} .
$$

Finally, we define the space

$$
W_{1, p}^{N L}\left(\Omega ; \mathbb{R}^{n}\right):=\left\{u \in L^{p}\left(\Omega ; \mathbb{R}^{n}\right): \nabla_{w}^{N L} u \in L^{p}\left(\Omega \times \Omega ; \mathbb{R}^{n}\right)\right\} .
$$

Assuming that the function $u$ is scalar-valued, the norm $\left\|\nabla_{w}^{N L} u\right\|_{L^{1}}$ corresponds to the anisotropic nonlocal total variation introduced by Gilboa and Osher [15], as well as the contrast modification term in the perceptual color correction model proposed by Bertalmío et al. [4].

Definition 3 (Adjoint of a nonlocal gradient operator). We define the adjoint of the operator $\nabla_{w}^{N L}$ as the operator $\nabla_{w}^{N L^{*}}: C^{\infty}\left(\Omega \times \Omega ; \mathbb{R}^{n}\right) \longrightarrow C^{\infty}\left(\Omega ; \mathbb{R}^{n}\right)$ satisfying

$$
\left\langle\nabla_{w}^{N L} u, \eta\right\rangle=\left(u, \nabla_{w}^{N L^{*}} \eta\right)
$$

where $($,$) is the L^{2}$ scalar product on $C^{\infty}\left(\Omega ; \mathbb{R}^{n}\right)$ induced by $h$. 
As in the scalar case in [15], a straightforward computation yields

$$
\nabla_{w}^{N L^{*}} \eta: x \longmapsto \int_{\Omega} w(x, y)(\eta(y, x)-\eta(x, y)) d y .
$$

We derive from Definition 3 the definition of nonlocal vectorial total variation.

Definition 4 (Nonlocal vectorial total variation). Let $u \in L^{1}\left(\Omega ; \mathbb{R}^{n}\right)$ and $w: \Omega \times \Omega \longrightarrow \mathbb{R}^{+*}$ be a smooth symmetric function. We define the nonlocal vectorial total variation $V T V_{w}^{N L}$ of $u$ as the quantity

$$
\sup _{\xi \in \mathcal{K}_{1}}\left(\int_{\Omega}(u(x), \xi(x))_{h} d x\right)
$$

where, for $a \in \mathbb{R}$,

$$
\mathcal{K}_{a}:=\left\{\nabla_{w}^{N L^{*}} \eta: \eta \in C^{\infty}\left(\Omega \times \Omega ; \mathbb{R}^{n}\right),\|\eta(x, y)\|_{h} \leq a \forall x, y \in \Omega\right\} .
$$

We denote by $B V_{w}^{N L}\left(\Omega, \mathbb{R}^{n}\right)$ the set of functions $u \in L^{1}\left(\Omega ; \mathbb{R}^{n}\right)$ such that $V T V_{w}^{N L}(u)<+\infty$.

Proposition 1. If $u \in W_{1,1}^{N L}\left(\Omega ; \mathbb{R}^{n}\right)$ then,

$$
V T V_{w}^{N L}(u)=\left\|\nabla_{w}^{N L} u\right\|_{L^{1}} .
$$

Proof. The proof is omitted for the sake of shortness.

Properties of $V T V_{w}^{N L}$ :

Let us first notice that $V T V_{w}^{N L}$ is a sup of linear forms

$$
J_{\eta}: u \longmapsto \int_{\Omega}\left(u(x), \nabla_{w}^{N L^{*}} \eta(x)\right)_{h} d x
$$

which are continuous with respect to the weak topology of $L^{2}\left(\Omega ; \mathbb{R}^{n}\right)$ since they are bounded. Hence, for $u \in L^{2}\left(\Omega, \mathbb{R}^{n}\right)$ and $u_{n} \rightarrow u$, we have $J_{\eta}\left(u_{n}\right) \rightarrow J_{\eta}(u)$. We then deduce that $V T V_{w}^{N L}$ is lower semi-continuous and convex.

\section{Dual principle of two nonlocal image processing models}

\subsection{Color image regularization}

In [15], Gilboa and Osher developed a nonlocal extension of the Rudin-OsherFatemi model [22] based on the anisotropic nonlocal total variation. More precisely, they proposed the following variational problem

$$
\underset{u}{\arg \min } \frac{\lambda}{2}\left\|u-u_{0}\right\|_{L^{2}}^{2}+\left\|\nabla_{w}^{N L} u\right\|_{L^{1}},
$$

and solved it numerically through a graph-cut technique.

The following proposition shows that we can express the solution of the model (6) assuming that it belongs to the space $L^{2} \cap B V_{w}^{N L}(\Omega ; \mathbb{R})$. We actually prove this result in the more general case of vector-valued functions. 
Proposition 2. The unique solution $\underline{u}$ of the variational problem

$$
\underset{u \in L^{2} \cap B V_{w}^{N L}\left(\Omega ; \mathbb{R}^{n}\right)}{\arg \min } E(u):=\frac{\lambda}{2}\left\|u-u_{0}\right\|_{L^{2}}^{2}+V T V_{w}^{N L}(u)
$$

is

$$
\underline{u}=u_{0}-P_{K_{\frac{1}{\lambda}}} u_{0}
$$

where $P$ is the projection operator, and $K_{\frac{1}{\lambda}}$ is the closure in $L^{2}\left(\Omega ; \mathbb{R}^{n}\right)$ of the set $\mathcal{K}_{\frac{1}{\lambda}}$ defined in (4).

Proof. The proof is a straightforward generalization of the proof in the case of the Rudin-Osher-Fatemi model whose details can be found in [12], and we omit it for the sake of shortness.

In the discrete case, the set $\mathcal{K}_{\frac{1}{\lambda}}$ is its own closure, and we have

$$
P_{\mathcal{K}_{\frac{1}{\lambda}}}\left(u_{0}\right)=\frac{1}{\lambda} \nabla_{w}^{N L^{*}} \underline{\eta}, \quad \text { where } \quad \underline{\eta}=\underset{\eta,\|\eta\|_{h} \leq 1}{\arg \min }\left\|\lambda u_{0}-\nabla_{w}^{N L^{*}} \eta\right\|_{L^{2}} .
$$

The Karush-Kuhn-Tucker conditions for the minimization problem

$$
\underset{\eta,\|\eta\|_{h} \leq 1}{\arg \min }\left\|\lambda u_{0}-\nabla_{w}^{N L^{*}} \eta\right\|_{L^{2}}
$$

are

$$
\left\{\begin{array}{l}
\nabla_{w}^{N L}\left(\nabla_{w}^{N L^{*}} \eta-\lambda u_{0}\right)_{i, j}+\alpha_{i, j} \eta_{i, j}=0 \\
\alpha_{i, j}\left(\left\|\eta_{i, j}\right\|_{h}-1\right)=0 \\
\alpha_{i, j} \geq 0
\end{array}\right.
$$

$\forall(i, j) \in \Omega$, from which follows that the Lagrange multipliers $\alpha_{i, j}$ satisfy

$$
\alpha_{i, j}=\left\|\nabla_{w}^{N L}\left(\nabla_{w}^{N L^{*}} \eta-\lambda u_{0}\right)_{i, j}\right\|_{h}
$$

We can then adapt Chambolle's projection algorithm in [11] to the minimization problem (9) and straightforward computations show that, if we consider the semi-implicit gradient descent

$$
\eta^{n+1}=\frac{\eta^{n}-d t \nabla_{w}^{N L}\left(\nabla_{w}^{N L^{*}} \eta^{n}-\lambda u_{0}\right)}{1+d t\left\|\nabla_{w}^{N L}\left(\nabla_{w}^{N L^{*}} \eta^{n}-\lambda u_{0}\right)\right\|_{h}}
$$

then the sequence $\frac{1}{\lambda} \nabla_{w}^{N L^{*}} \eta^{n}$ converges towards $P_{\mathcal{K}_{1 / \lambda}} u_{0}$, assuming that $d t \leq$ $1 /\left\|\nabla_{w}^{N L^{*}}\right\|^{2}$.

\subsection{Perceptual color correction}

In [4], Bertalmío et al. propose the following variational model for performing perceptual correction of a color image $u_{0}=\left(u_{0}^{1}, u_{0}^{2}, u_{0}^{3}\right)$

$$
\underset{u^{k}}{\arg \min } \frac{\lambda}{2}\left\|u^{k}-u_{0}^{k}\right\|_{L^{2}}^{2}+\frac{\beta}{2}\left\|u^{k}-1 / 2\right\|_{L^{2}}^{2}-\left\|\nabla_{w}^{N L} u^{k}\right\|_{L^{1}}, \quad k=1,2,3
$$


They showed that the problem (12) has a solution in the discrete case. In order to construct a solution, they considered a regularized version of the problem (12) by regularizing the anisotropic total variation $\left\|\nabla_{w}^{N L} u^{k}\right\|_{L^{1}}$ and performed a gradient descent until reaching a steady-state.

In the following proposition, we show that we can express the solutions of the model (12) in the discrete case by assuming that they belong to the discrete space $L^{2} \cap B V_{w}^{N L}(\Omega ; \mathbb{R})$. We actually prove this result in the more general case of vector-valued functions.

Proposition 3. Let $X$ be the discrete space $L^{2} \cap B V_{w}^{N L}\left(\Omega ; \mathbb{R}^{n}\right)$, and $u_{0}, v \in X$. The solutions of the variational problem

$$
\underset{u \in X}{\arg \min } \frac{\lambda}{2}\left\|u-u_{0}\right\|_{L^{2}}^{2}+\frac{\beta}{2}\|u-v\|_{L^{2}}^{2}-V T V_{w}^{N L}(u)
$$

are

$$
\underline{u}=\frac{1}{\lambda+\beta}\left(\lambda u_{0}+\beta v\right)-\underset{u^{\star} \in \mathcal{K} \frac{1}{\lambda+\beta}}{\arg \max }\left\|\frac{1}{\lambda+\beta}\left(\lambda u_{0}+\beta v\right)-u^{\star}\right\|_{L^{2}}^{2},
$$

where $\mathcal{K}_{\frac{1}{\lambda+\beta}}$ is the discretization of the space $\mathcal{K}_{\frac{1}{\lambda+\beta}}$ defined in (4).

Proof. Let us first observe that the problem (13) is equivalent to

$$
\underset{u \in X}{\arg \min } \frac{\lambda+\beta}{2}\left\|u-\frac{1}{\lambda+\beta}\left(\lambda u_{0}+\beta v\right)\right\|_{L^{2}}^{2}-V T V_{w}^{N L}(u) .
$$

The problem (15) is of the form

$$
\inf _{u \in V}\{G(u)-F(u)\}
$$

where $F$ and $G$ are two lower semi-continuous functionals on a reflexive Banach space $V$ such that $F$ is convex and $G$ satisfies $G(u) /\|u\| \rightarrow \infty$ as $\|u\| \rightarrow \infty$. Then according to Theorem 2.7 in [24], if $\underline{u}^{*}$ is a solution of the dual problem

$$
\inf _{u^{*} \in V^{*}}\left\{F^{*}\left(u^{*}\right)-G^{*}\left(u^{*}\right)\right\} \text {, }
$$

then there exists a solution $\underline{u}$ of the primal problem (16), and the solutions of both problems are connected by the formulas

$$
\begin{aligned}
& F(\underline{\mathrm{u}})+F^{*}\left(\underline{\mathrm{u}}^{*}\right)=\left(\underline{\mathrm{u}}, \underline{\mathrm{u}}^{*}\right) \\
& G(\underline{\mathrm{u}})+G^{*}\left(\underline{\mathrm{u}}^{*}\right)=\left(\underline{\mathrm{u}}, \underline{\mathrm{u}}^{*}\right) .
\end{aligned}
$$

Hence, the problem (13) can be solved through its dual problem (17).

The convex conjugate of the functional

$$
G: u \longmapsto \frac{\lambda+\beta}{2}\left\|u-\frac{1}{\lambda+\beta}\left(\lambda u_{0}+\beta v\right)\right\|_{L^{2}}^{2}
$$


is

$$
G^{*}: u^{*} \longmapsto \frac{1}{2(\lambda+\beta)}\left[\left\|u^{*}\right\|_{L^{2}}^{2}+2\left(u^{*}, \lambda u_{0}+\beta v\right)\right] .
$$

The functional $F:=V T V_{w}^{N L}$ being one homogeneous, proper, lower semicontinuous and convex, its convex conjugate $F^{*}$ is the indicator function $\chi_{\mathcal{K}_{1}}$ of the set $\mathcal{K}_{1}$, which is the discretization of the set $\mathcal{K}_{1}$ defined in (4). Then, the dual problem (17) reads

$$
\underset{u^{*} \in X}{\arg \min } \chi_{\mathcal{K}_{1}}\left(u^{*}\right)-\frac{1}{2(\lambda+\beta)}\left[\left\|u^{*}\right\|_{L^{2}}^{2}+2\left(u^{*}, \lambda u_{0}+\beta v\right)\right] .
$$

The functional (21) is bounded from below and coercive since $X$ is a subspace of the discrete space $L^{2}\left(\Omega ; \mathbb{R}^{n}\right)$, and Theorem 2.9 in [24] guarantees then that the problem $(21)$ has a solution $\underline{u}^{*}$. The dual problem can then be rewritten

$$
\underline{u}^{*}=\underset{u^{*} \in \mathcal{K}_{1}}{\arg \max }\left\|u^{*}\right\|_{L^{2}}^{2}+2\left(u^{*}, \lambda u_{0}+\beta v\right) .
$$

Modifying the coefficients of the terms $\left\|u_{0}\right\|$ and $\|v\|$ does not affect the problem (22), hence we can write

$$
\underline{u}^{*}=\underset{u^{*} \in \mathcal{K}_{1}}{\arg \max }\left\|u^{*}\right\|_{L^{2}}^{2}+2\left(u^{*}, \lambda u_{0}+\beta v\right)+\left\|\lambda u_{0}+\beta v_{0}\right\|_{L^{2}}^{2}
$$

i.e.

$$
\underline{u}^{*}=\underset{u^{*} \in \mathcal{K}_{1}}{\arg \max }\left\|\lambda u_{0}+\beta v+u^{*}\right\|_{L^{2}}^{2}
$$

which is equivalent to

$$
\underline{u}^{*}=\underset{u^{*} \in \mathcal{K}_{1}}{\arg \max }\left\|\lambda u_{0}+\beta v-u^{*}\right\|_{L^{2}}^{2} .
$$

Finally, we deduce from (19) and (20) that the solutions $\underline{u}$ of the original problem (13) are

$$
\underline{u}=\frac{1}{\lambda+\beta}\left[\lambda u_{0}+\beta v+\underline{u}^{*}\right],
$$

which is equivalent to the expression (14).

We have

$\underset{u^{*} \in \mathcal{K}_{1}}{\arg \max }\left\|\lambda u_{0}+\beta v-u^{*}\right\|_{L^{2}}^{2}=\nabla_{w}^{N L^{*}} \underline{\eta}$, where $\underline{\eta}=\underset{\eta,\|\eta\|_{h} \leq 1}{\arg \max }\left\|\lambda u_{0}+\beta v-\nabla_{w}^{N L^{*}} \eta\right\|_{L^{2}}$.

The Karush-Kuhn-Tucker conditions for the maximization problem

$$
\underset{\eta,\|\eta\|_{h} \leq 1}{\arg \max }\left\|\lambda u_{0}+\beta v-\nabla_{w}^{N L^{*}} \eta\right\|_{L^{2}}
$$

are

$$
\left\{\begin{array}{l}
\nabla_{w}^{N L}\left(\nabla_{w}^{N L^{*}} \eta-\left(\lambda u_{0}+\beta v\right)\right)_{i, j}-\alpha_{i, j} \eta_{i, j}=0 \\
\alpha_{i, j}\left(\left\|\eta_{i, j}\right\|_{h}-1\right)=0 \\
\alpha_{i, j} \geq 0
\end{array}\right.
$$


$\forall(i, j) \in \Omega$, from which follows that the Lagrange multipliers $\alpha_{i, j}$ satisfy

$$
\alpha_{i, j}=\left\|\nabla_{w}^{N L}\left(\nabla_{w}^{N L^{*}} \eta-\left(\lambda u_{0}+\beta v\right)\right)_{i, j}\right\|_{h} .
$$

We deduce from the Karush-Kuhn-Tucker conditions (28) that we can adapt Chambolle's projection algorithm in [11] to the maximization problem (27): we consider the following semi-implicit gradient ascent

$$
\eta^{n+1}=\frac{\eta^{n}+d t \nabla_{w}^{N L}\left(\nabla_{w}^{N L^{*}} \eta^{n}-\left(\lambda u_{0}+\beta v\right)\right)}{1+d t\left\|\nabla_{w}^{N L}\left(\nabla_{w}^{N L^{*}} \eta^{n}-\left(\lambda u_{0}+\beta v\right)\right)\right\|_{h}},
$$

and a computation similar to the one done in the proof of Theorem 3.1.in [11] shows that the sequence $\left\|\lambda u_{0}+\beta v-\nabla_{w}^{N L^{*}} \eta^{n}\right\|$ is strictly increasing with $n$ unless the sequence (29) reaches a fixed point $\eta$, which is either a local or global maximum. Indeed, we have

$$
\eta^{n+1}=\eta^{n} \Longrightarrow \nabla_{w}^{N L}\left(\nabla_{w}^{N L^{*}} \eta-\left(\lambda u_{0}+\beta v\right)\right)_{i, j}-\alpha_{i, j} \eta_{i, j}=0 \quad \forall(i, j) \in \Omega
$$

and unlike minima, local maxima of convex functions are not necessarily global.

It can also be shown that any converging subsequence $\eta^{n_{k}}$ of $\eta^{n}$ converges towards a fixed point, i.e. a local maximum. Hence, the sequence $\eta^{n}$ possesses local maxima as adherent points.

\section{A new insight on the two variational problems}

\subsection{Duality principle for a reinterpretation of the models}

We showed in section 3.1 that the regularization model of Gilboa and Osher [15] based on the so-called anisotropic nonlocal total variation has also a dual formulation, that yields the following expression of its unique solution $\underline{u}_{R}$ :

$$
\underline{u}_{R}=u_{0}-\underset{u^{\star} \in K_{1 / \lambda}}{\arg \min }\left\|u_{0}-u^{\star}\right\|_{L^{2}}^{2},
$$

where the functional space $K_{1 / \lambda}$ is the closure of the space $\mathcal{K}_{1 / \lambda}$ defined in (4). The spaces $\mathcal{K}_{1 / \lambda}$, for $\lambda \in \mathbb{R}$, can be viewed as subspaces of the space

$$
G_{w}^{N L}:=\left\{\nabla_{w}^{N L^{*}} \eta: \eta \in L^{\infty}\left(\Omega \times \Omega ; \mathbb{R}^{n}\right)\right\},
$$

which has been introduced by Gilboa and Osher [15] in the scalar case, as a nonlocal extension of the Meyer's space of oscillating patterns [19]. They interpret the space $G_{w}^{N L}$ as characterizing irregular and random (scalar-valued) patterns. Assuming that the conjecture is true, our dual formulation (30) provides a better understanding of the original model: it consists in removing the main irregular and random patterns of the image $u_{0}$, the measure of irregularity and randomness being given by both the scalar $\lambda$ and the function $w$.

Note that this conclusion had already been provided by Gilboa and Osher 
when the anisotropic nonlocal total variation in the regularization model is replaced by the isotropic nonlocal total variation. We then deduce that the difference between the two regularization models holds in the measure of irregularity and randomness of the patterns.

We showed in section 3.2 that the discrete perceptual color correction model of Bertalmío et al. (12) possesses a dual formulation too, from which derive a novel expression of its solutions $\underline{u}_{C E}=\left(\underline{u}_{C E}^{1}, \underline{u}_{C E}^{2}, \underline{u}_{C E}^{3}\right)$, given, for $k=1,2,3$, by

$$
\underline{u}_{C E}^{k}=\frac{1}{\lambda+\beta}\left(\lambda u_{0}^{k}+\beta / 2\right)-\underset{u^{\star} \in K_{\frac{1}{\lambda+\beta}}}{\arg \max }\left\|\frac{1}{\lambda+\beta}\left(\lambda u_{0}^{k}+\beta / 2\right)-u^{\star}\right\|_{L^{2}}^{2},
$$

where $K_{\frac{1}{\lambda+\beta}}$ denotes here the discretization of the space $\mathcal{K}_{\frac{1}{\lambda+\beta}}$ defined in (4). We then deduce that the model (12) can be decomposed into two steps:

1. It first performs global color constancy of the original image $u_{0}=\left(u_{0}^{1}, u_{0}^{2}, u_{0}^{3}\right)$ through the functions

$$
\frac{1}{\lambda+\beta}\left(\lambda u_{0}^{k}+\beta / 2\right), \quad k=1,2,3 .
$$

Indeed, a straightforward computation shows that $\left(\lambda u_{0}^{k}+\beta / 2\right) /(\lambda+\beta)$ is closer to $1 / 2$ than $u_{0}^{k}, \forall k \in\{1,2,3\}$, meaning that the color cast of $u_{0}$ has been reduced (see [20] for details).

2. It performs contrast enhancement of the color corrected image (33) by removing the most distant element in the space $K_{\frac{1}{\lambda+\beta}}$ of each of its channel, given by

$$
\underset{u^{\star} \in K \frac{1}{\lambda+\beta}}{\arg \max }\left\|\frac{1}{\lambda+\beta}\left(\lambda u_{0}^{k}+\beta / 2\right)-u^{\star}\right\|_{L^{2}}^{2}, \quad k=1.2 .3,
$$

where $K_{\frac{1}{\alpha+\beta}}$ has been interpreted above as a subspace of a nonlocal extension of the Meyer's space of oscillating patterns.

\subsection{Applications}

We apply on a color image (Fig. 1(a)) the proposed vectorial extensions of the regularization model of Gilboa and Osher [15], whose unique solution is given in (8), and the perceptual color correction model of Bertalmío et al. [4], whose solutions are given in (14). On this latter model, we have set the constant $\beta$ to 0 , i.e. we reduce the model to enhance the contrast of the original image. Then, taking the same values for the other parameters, both models only differ by the sign of the nonlocal total variation, and their solutions only differ by the element taken in the discrete set $K_{\frac{1}{\lambda}}$, whose continuous version is defined in (4). 
In the experiment we perform, the parameter $\lambda$ has been set to 0.05 , and the definite positive quadratic form $h$ is nothing but the Euclidean metric in the $R G B$ color space. The symmetric positive function $w$ has been chosen as the 2D Gaussian kernel of variance 2000 that we have truncated by setting the values $w(x, y)$ to 0 if $y$ is not in the $15 \times 15$ neighborhood of $x$, the aim of this procedure being to reduce the complexity of the algorithm. We use the same stopping criteria for both algorithms (11) and (29), which is

$$
\left\|\eta^{n+1}-\eta^{n}\right\|_{L^{2}}<0.001
$$

Results are respectively shown on Fig. 1(b) and Fig. 1(c). We also show the differences between the results and the original image, which are elements of the discrete set $K_{\frac{1}{\lambda}}$. In both models, we observe that the negative part (Fig. 1(d,e)) tends to vanish where the positive part (Fig. 1(f,g)) does not vanish and vice versa. We also observe that the negative component in the regularization model behaves like the positive component in the contrast enhancement model and vice versa, which is coherent with the fact that the models only differ by the sign of the anisotropic nonlocal total variation. Note that by our choice of the parameter $w$, the regularization model is acting as a contrast reduction model, which is actually the vectorial extension of the model used by Zamir et al. [25] for color gamut reduction purpose.

Finally, we would like to point out that the contrast enhancement model can produce values out of the range $[0,255]$ if the parameter $\lambda$ is taken too small.

\section{Conclusion}

In this paper, we have constructed dual formulations of two existing image processing variational models, namely the image regularization model of Gilboa and Osher [15] and the perceptual color correction model of Bertalmío et al. [4]. Using these dual formulations, we have been able to reinterpret their solutions, providing a new insight on these models.

Further work will be devoted to investigate whether the nonlocal space (4) that came out from our dual formulations has a perceptual interpretation. Moreover, inspired by the local case with the work of Meyer [19] on the space of oscillating patterns, we expect that there exists a norm on the space (4) that would yield a better perceptual color correction than the L2 norm in (34).

\section{References}

1. Awate, S.P., Whitaker, R.T.: Unsupervised, information-theoretic, adaptive image filtering for image restoration. IEEE Trans. Pattern Anal. Mach. Intell. 28(3), 364$376(2006)$

2. Batard, T., Bertalmío, M.: On covariant derivatives and their applications to image regularization. SIAM J. Imaging Sci. 7(4), 2393-2422 (2014)

3. Bertalmío, M.: Image Processing for Cinema. Chapman \& Hall/CRC 
4. Bertalmío, M., Casselles, V., Provenzi, E., Rizzi, A.: Perceptual color correction through variational techniques. IEEE Trans. Im. Processing 16(4), 1058-1072 (2007)

5. Bertalmío, M., Caselles, V., Provenzi, E.: Issues about Retinex theory and contrast enhancement. Int. J. Computer Vision 83, 101-119 (2009)

6. Bertalmío, M., Cowan, J.D.: Implementing the Retinex algorithm with WilsonCowan equations. J. Physiology 103, 69-72 (2009)

7. Bredies, K., Kunish, K., Pock, T.: Total generalized variation. SIAM J. Imaging Sci., 3(3) (2010), pp. 492-526.

8. Bresson, X., Chan, T. F.: Fast dual minimization of the vectorial total variation norm and applications to color image processing. Inverse Probl. Imaging 2(4), 455$484(2008)$

9. Buades, A., Coll, B., Morel, J.-M.: A non-local algorithm for image denoising. Proceedings of CVPR (2), 60-65 (2005)

10. Buchsbaum, G.: A spatial processor model for object color perception. J. Franklin Inst., 310(1), 1-26 (1980)

11. Chambolle, A.: An algorithm for total variation minimization and applications. J. Math. Imaging Vision 20, 89-97 (2004)

12. Chambolle, A., Caselles, V., Cremers, D., Novaga, M., Pock, T.: An introduction to total variation for image analysis. Theoretical Foundations and Numerical Methods for Sparse Recovery 9, 263-340 (2010)

13. http://rit-mcsl.org/fairchild/HDR.html

14. Ferradans, S., Bertalmío, M., Provenzi, E., Caselles, V.: An analysis of visual adaptation and contrast perception for tone mapping. IEEE Trans. Pattern Anal. Mach. Intell. 33(10), pp. 2002-2012 (2011)

15. Gilboa, G., Osher, S.: Nonlocal operators with applications to image processing. Multiscale Model. Simul. 7(3), 1005-1028 (2008)

16. Goldluecke, B., Strekalovskiy, E., Cremers, D.: The natural vectorial total variation which arises from geometric measure theory. SIAM J. Imaging Sci. 5, 537-563 (2012)

17. Jin, Y., Jost, J., Wang, G.: A new nonlocal $H^{1}$ model for image denoising. J. Math. Imaging Vision 48(1), 93-105 (2014)

18. Kindermann, S., Osher, S., Jones, P.W.: Deblurring and denoising of images by nonlocal functionals. Multiscale Model. Simul. , 1091-1115 (2005)

19. Meyer, Y.: Oscillating Patterns in Image Processing and in some Nonlinear Evolution Equations. The Fifteenth Dean Jacqueline B. Lewis Memorial Lectures (2001).

20. Palma-Amestoy, R., Provenzi, E., Bertalmío, M., Caselles, V.: A perceptually inspired variational framework for color enhancement. IEEE Trans. Pattern Anal. Mach. Intell. 31(3), 458-474 (2009)

21. Rockafellar, R. T.: Convex Analysis. Princeton University Press (1970)

22. Rudin, L.I., Osher, S., Fatemi, E.: Nonlinear total variation based noise removal algorithms. Physica D 60, 259-268 (1992)

23. Sapiro, G., Caselles, V.: Histogram modification via differential equations. J. Differential Equations 135, 238-268 (1997)

24. Toland, J.F.: A duality principle for non-convex optimisation and the calculus of variations. Archiv. Rational Mech. Analysis 71(1), 41-61 (1979)

25. Zamir, S. W., Vazquez-Corral, J., Bertalmío, M.: Gamut mapping in cinematography through perceptually-based contrast modification. J. Sel. Topics Signal Processing 8(3), 490-503 (2014).

26. Zosso, D., Tran, G., Osher, S.: Non-local Retinex- A unifying framework and beyond. To appear in SIAM J. Imaging Sci. 


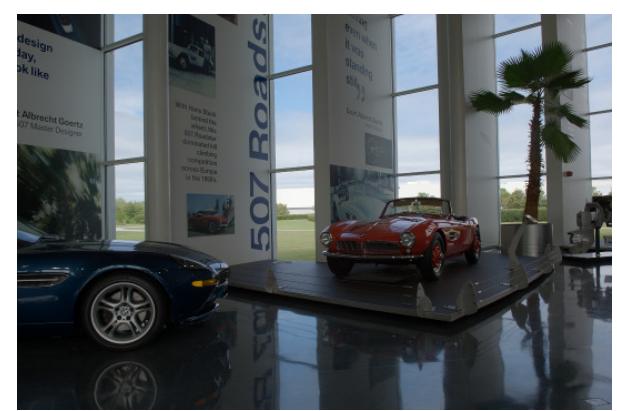

(a) Image $u_{0}$ (tone-mapped version of the high dynamic range image "507" in the database [13])

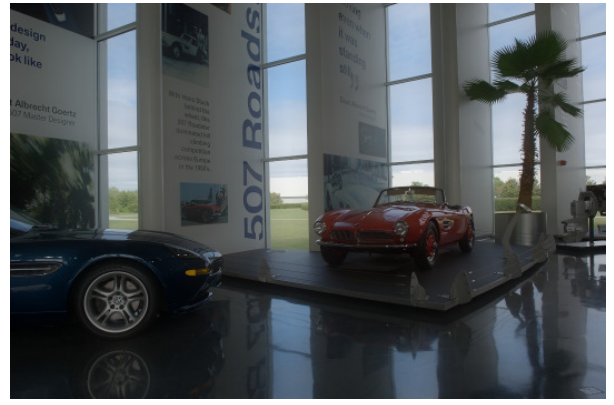

(b) Regularization $\underline{u}_{R}$ of $u_{0}$

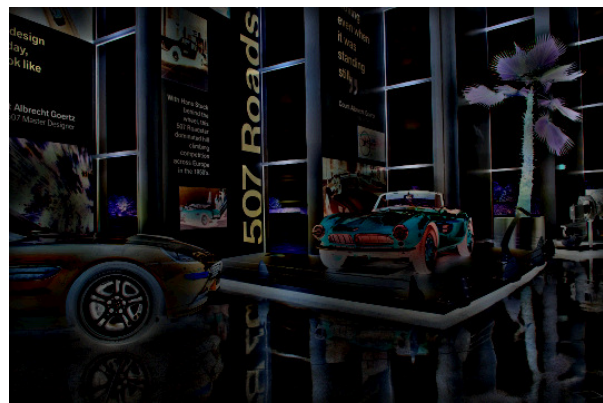

(d) Negative component of $u_{0}-\underline{u}_{R}$

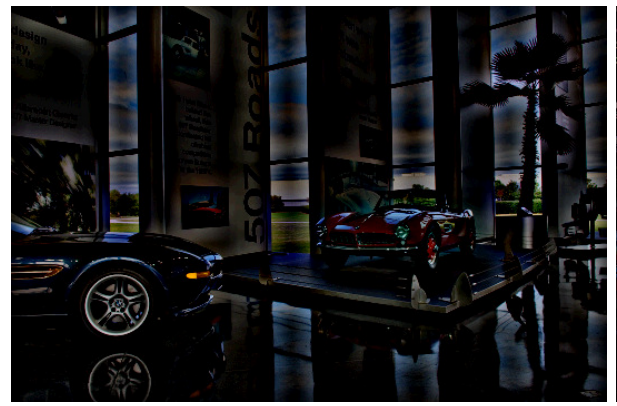

(f) Positive component of $u_{0}-\underline{u}_{R}$

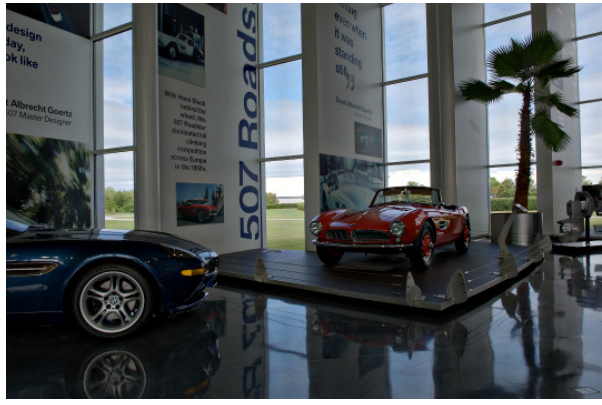

(c) Contrast enhancement $\underline{u}_{C E}$ of $u_{0}$

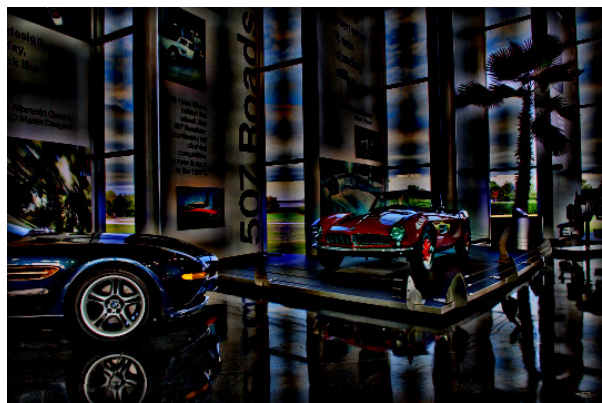

(e) Negative component of $u_{0}-\underline{u}_{C E}$

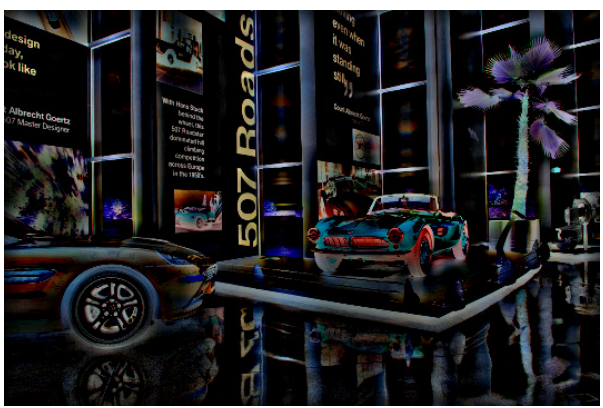

(g) Positive component of $u_{0}-\underline{u}_{C E}$

Fig. 1. Comparison between the proposed vectorial extensions $\underline{u}_{R}$ of the regularization model of Gilboa and Osher [15] and $\underline{u}_{C E}$ of the second step (contrast enhancement) of the perceptual color correction model of Bertalmío et al. [4]. Intensity values of the figures (d),(e),(f),(g) have been multiplied by 10 in order to improve the visibility. 